\title{
Zur nacheiszeitlichen Gletschergeschichte des Liefdefjords (Spitzbergen)* (Ergebnisse der Geowissenschaftlichen Spitzbergenexpedition 1990)
}

\section{Introduction and Summary}

The region of the Liefdefjord in Northern Spitsbergen $\left(79^{1} / 2^{\circ}\right.$ $\mathrm{N})$ was the study area on an international and interdisciplinary working Spitsbergen expedition during summer 1990 and again in 1991. The main research objectives were focussed primarily on the holocene and recent morphologic and geoecological processes in a territory early untouched by human activities. The area visited by this expedition is characterised by numerous cirque- and valley glaciers as well as by "alpine" like types of landforms.

The results of the subproject "Glacial History and Dating of Morainic Sequences" presented in this paper contribute to the glacial history of Spitsbergen, which is only partially known at the moment:

- Numerous glacier fronts (for example Monacobreen) are either reaching directly into the sea or are ending in a position close to the shoreline. The glacier forefields are most often surrounded by very distinct but rather complex morainic systems. The morainic deposits adjacent to the recent glacier margins are built up typically by Neoglacial or Little Ice Age advances (since the 16th. century).

- In the investigation area of Liefdefjorden and Bockfjorden numerous fossil organic soil horizons ( $f A h$ ) could be found by digging through the leeward side of the morainic ridges. These soils are interpreted to be overridden and buried by former glacial advances. The radiocarbon method reveals different glacier advances dating of the early and late phases of the Older Subatlantic (2800-1000 yBP) and of the early and middle phases of the Younger Subatlantic (since $1000 \mathrm{yBP}$ ).

- Thickness and pollen content of the dated fossil soil horizons and the peat bog layers suggest a strong similarity to the present vegetation cover close to the coastline. Therefore the climatic conditions during the soil and peat bog formation were at least and most likely not less favorable than they are today.

- Consequently these results lead to the conclusion, that the morainic systems contouring the boundaries of the recent glacier forefields consist of material resulting from several advances. They represent similar extensions but different ages. Since 2800 yBP (probably during the entire Holocene) the glacier variations and their maximum extensions must have been oscillating always within the same limits. These two characteristics are particularly typical also for the holocene glacial history found in the Alps.

- Direct evidence of early holocene or lateglacial advances could not be found in the study area. The question concerning the pattern of glacier behavior during the Younger Dryas (11000-10 $200 \mathrm{yBP})$ is therefore still unanswered. Based on our field observations we suppose that the ice margins during these older glacial periods possibly did not exceed the outermost limits of the Subatlantic moraines.
- The region of the Liefdefjord was deglaciated prior to the beginning of the Alleröd period (11800-11000 yBP) and the glaciers then reached at least an extent similar to the recent one.

\section{Einleitung und Zusammenfassung}

Der Liefdefjord in Nordwestspitzbergen $\left(79 \%_{2}^{\circ} \mathrm{N}\right)$ war 1990 - und 1991 nochmals - das Arbeitsgebiet der internationalen, interdisziplinären Spitzbergenexpedition SPE '90. Schwerpunkte der Forschungsarbeiten sind nacheiszeitliche und aktuelle geomorphologische und geoökologische Prozesse in diesem vom Menschen noch fast völlig unberührten Raum. Das Expeditionsgebiet besitzt zahlreiche Kar- und einzelne Talgletscher sowie ein stark differenziertes, «alpin» anmutendes Relief.

Mit unserem Teilprojekt «Gletscherentwicklung und Moränendatierung» wird versucht, einen Beitrag zur noch wenig bekannten Gletschergeschichte Spitzbergens zu leisten:

- Viele Gletscher - so der Monacobreen - münden heute direkt ins Meer oder enden nahe der Küste. Die

* Der Schweizerische Nationalfonds zur Förderung der wissenschaftlichen Forschung hat unsere Teilnahme an dieser von der Deutschen Forschungsgemeinschaft getragenen Expedition ermöglicht. Die für die Altersbestimmung erforderliche Aufbereitung des Probenmaterials erfolgte im Radiokarbonlabor des Geographischen Institutes der Universität Zürich. Die anschließende Datierung wurde mittels der AMS-Technik (accelerator mass spectrometry) auf dem Tandem-Beschleuniger des IMP (Institut für Mittelenergiephysik) der ETH-Hönggerberg durchgeführt und dank finanzieller Unterstützung durch die Stiftung für wissenschaftliche Forschung an der Universität Zürich ermöglicht.

Gerhard Furrer, Prof., Dr., und André Stapfer, Geographisches Institut der Universität Zürich, Winterthurerstraße 190, CH8057 Zürich/Ulrich Glaser, Dr., Geographisches Institut der Universität Würzburg, Am Hubland, D-8700 Würzburg. 
Gletschervorfelder sind in der Regel durch einen markanten Wallmoränenkomplex (Moränenwall) umschlossen. Das Moränenmaterial im gletschernahen Bereich wurde von Vorstößen der Neuzeit (ab 16. Jahrhundert) abgelagert.

- Im Liefdefjorden und Bockfjorden konnten aber durch Grabungen an der Außenseite der Moränenwälle mehrere, von Gletschern überfahrene, fossile organische Bodenhorizonte (fAh) gefunden werden. Deren Radiokarbondatierungen lassen auf Gletschervorstöße im frühen und späten Älteren Subatlantikum (2800-1000 yBP) und im frühen und mittleren Jüngeren Subatlantikum (ab 1000 yBP) schließen.

- Mächtigkeit und Polleninhalt der datierten fossilen Boden- und Torfbildungen deuten darauf hin, daß die Vegetation zur Zeit der organischen Sedimentation ähnlich wie heute in Küstennähe entwickelt war. Die klimatischen Bedingungen dürften während der früheren Torf-/Bodenbildungsphasen zumindest nicht ungünstiger gewesen sein als heute.
- Diese Resultate führen zur Schlußfolgerung, daß die heutigen Gletschervorfelder von Moränenwällen umschlossen sind, die aus Material von verschiedenen Gletschervorstößen zusammengesetzt sind, und daß die maximale Ausdehnung der Gletscherhochstände in den letzten 2800 Jahren (vermutlich im ganzen Holozän) stets etwa dieselbe war. Diese Charakteristika treffen auch für die nacheiszeitliche Gletschergeschichte der Alpen zu.

- Im Untersuchungsgebiet konnten keine Hinweise auf ältere postglaziale und spätglaziale Gletschervorstöße gefunden werden. Vor allem die Frage nach dem Gletscherverhalten während der Jüngeren Dryas (11 000-10200 yBP) bleibt offen. Aufgrund unserer Beobachtungen muß man vermuten, daß allfällige Gletschervorstöße während dieser Zeit die Maximalstände des Subatlantikums nicht übertroffen haben.

- Spätestens zu Beginn des Alleröds (11800-11000 yBP) war der Liefdefjord mindestens soweit eisfrei wie heute.

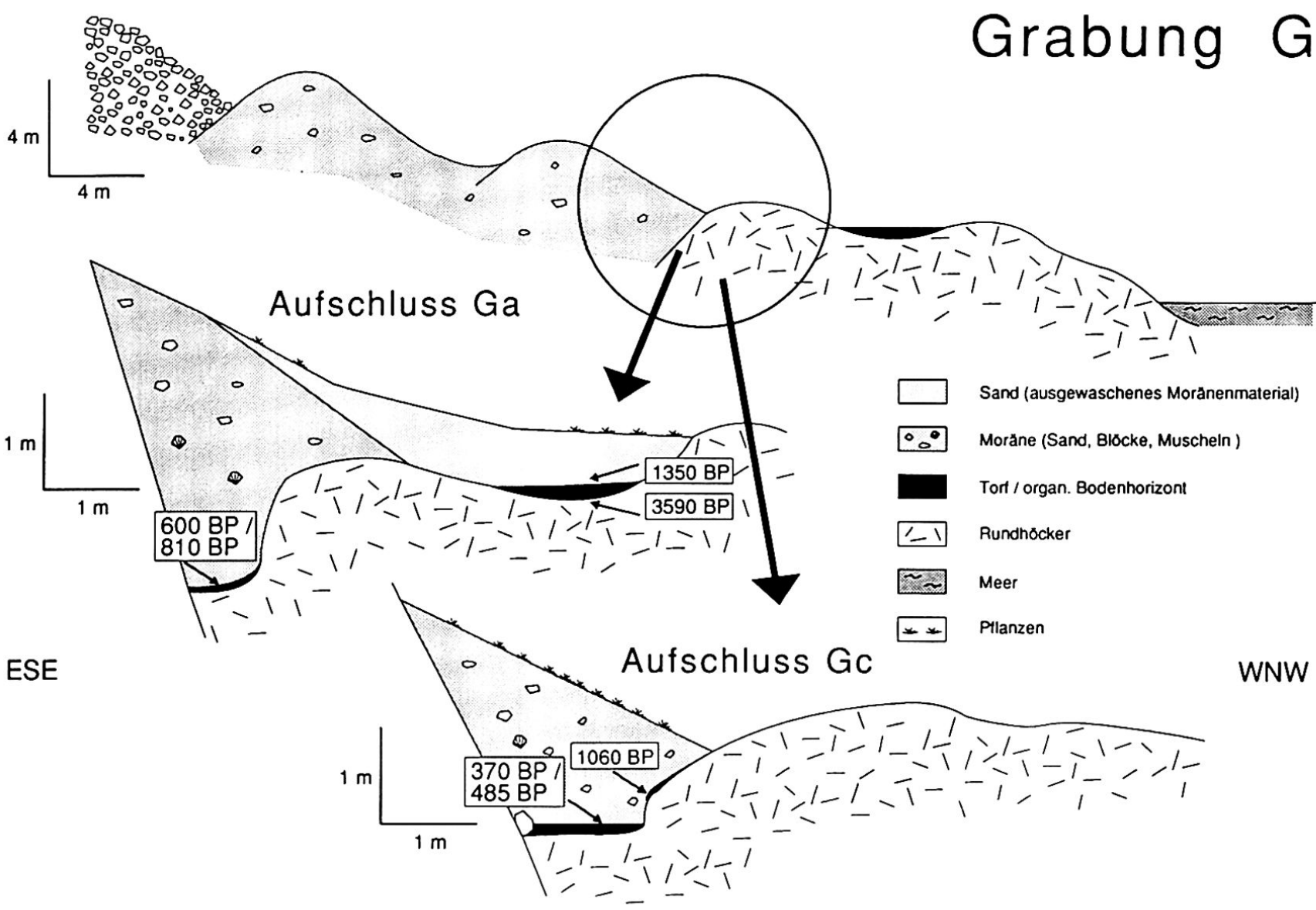

Fig. 1: Schnitt durch den Moränenwall des Glopbreen, wo dieser an einen Rundhöcker geschoben ist. Die beiden gegrabenen Aufschlüsse Ga und Gc (untere Skizzen) liegen rund $15 \mathrm{~m}$ auseinander, und die Grabungsstelle Gc liegt rund 0,5 m tiefer als Ga. Von der äußersten Moräne (Ga) ist schluffig-sandiges Material abgeschwemmt worden, es bedeckt eine $18 \mathrm{~cm}$ mächtige Torflinse in einer Wanne des Rundhöckers. 


\section{Der Glopbreen und seine Wallmoränen}

Auf seiner orographisch linken Seite, wo der Endmoränenwall in die Seitenmoräne übergeht, können drei dicht hintereinander liegende Wallmoränen beobachtet werden (Fig. 1). Dieser mehrgliedrige Moränenwall säumt das Gletschervorfeld, in dem zurückgestaffelt weitere Wälle bzw. Wallabschnitte entwickelt sind, die sich zu zwei inneren Ständen verbinden lassen. Die Kämme der drei erwähnten Wälle, welche den das Gletschervorfeld säumenden Moränenwall bilden, sind verschieden hoch. Ihre Höhenlage nimmt von außen nach innen zu, die Wälle unterscheiden sich außerdem in ihrem Material: Am Aufbau des äußersten ist besonders zusammengeschobenes Lagunenmaterial aus dem Gletschervorfeld beteiligt (Muscheln belegen marines Milieu), der höchste dagegen ist außerordentlich blockreich, während der mittlere Wall wenige Blöcke enthält, kantige und kantengerundete Steine sowie viel Feinmaterial, aber kaum Muscheln. Obwohl die drei Wallmoränen nicht durch fossile Böden voneinander getrennt sind, führen wir sie aufgrund der beschriebenen morphographischen und materialmäßigen Unterschiede auf drei verschieden alte Gletschervorstöße zurück. Am Glopbreen mit den zwei erwähnten intern gelegenen Ständen sind somit fünf Moränen nachweisbar.

Mit Hilfe von Grabungen (Fig. 2) gelang uns auch an andern Gletschern der Nachweis von fossilem organischem Material (FURRER, 1991b). Torfbildungen bzw. Humushorizonte treten oft aufFels unter Moränenmaterial und unter Wallmoränen in situ auf. Deshalb sind Grabungen in den Außenseiten von Wallmoränen besonders dort lohnend, wo diese an Rundhöcker herangeschoben wurden (Fig. 1). Wo zwei Wälle aneinanderstoßen, konnte dagegen kein organisches Material - etwa im Sinne eines fossilen Bodens (FURRER, 1991a) - zwischen ihnen beobachtet werden. Leider wurden die Grabungsarbeiten, die zu künstlichen Aufschlüssen führten, durch den Permafrost und in die Grabung einströmendes Wasser behindert. Im Sommer 1990 kam uns aber die Witterung entgegen: Im Basislager, fast auf Meereshöhe, sanken die Temperaturen nur selten unter den Nullpunkt, und die Tagesmitteltemperaturen lagen während unserer Expedition (vom 8. Juli bis 28. August) mehrheitlich zwischen $3^{\circ}$ und $8^{\circ} \mathrm{C}$. Die Tagesmaxima erreichte von Mitte Juli bis 4. August mehrheitlich Werte von über $10^{\circ} \mathrm{C}$ und stiegen gelegentlich auf über $12^{\circ} \mathrm{C}$. Unsere mit Pickel und Schaufel niedergebrachten Grabungen erstreckten sich daher in der Regel über mehrere Wochen: Sobald ein Weiterkommen im gefrorenen Boden nicht mehr möglich war, wurden die Grabarbeiten für einige Tage unterbrochen. Während diesen Unterbrechungen taute der Boden weiter nach der Tiefe auf. So gab der Permafrost mit der Zeit die organischen Ablagerungen frei.

\section{Interpretation der beiden Grabungen Ga und Gc}

Im Aufschluß Ga sind in der Moräne deutliche Stauchungserscheinungen zu beobachten. Steine liegen be- sonders nahe der Moränenoberfläche sowie auf ihr, im Innern der Moräne dagegen treten sie gegenüber dem Feinmaterial stark zurück. Nur selten finden sich in der Moräne Blöcke von rund $1 \mathrm{~m}$ Durchmesser und kantengerundete, etwa faustgroße Steine. Im Feinmaterial sind Muschelschalen und gelegentlich punktförmige schwarze, organische Materialien, in der Regel nur wenige Millimeter groß, eingebettet. Beim Graben trifft man - allerdings ganz selten - auf etwas größere organische Reste, beispielsweise auf Moose (bestimmt wurde die Art Dicranum angustum) und auf abgestorbene Pflanzenwurzeln. Unter den Muschelschalen ist besonders die heute boreal bis arktisch verbreitete Mya truncata vertreten. Das verstreut auftretende pflanzliche Material muß beim Gletschervorstoß im Gletschervorfeld abgetragen und mit der Moränenbildung akkumuliert worden sein. Die in die Moräne eingebetteten Muschelschalen deuten darauf hin, daß Teile des Gletschervorfeldes (zeitweise) unter dem Meeresspiegel lagen und marines Material am Moränenaufbau beteiligt ist.

Die Mächtigkeit der Torflinse im Aufschluß Ga, die auBerhalb der eigentlichen Wallmoräne auf dem Rundhökker fossilisiert wurde, beträgt $18 \mathrm{~cm}$. An ihrer Basis liegen einige Splitter aus dem anstehenden Fels (Rundhöcker in ihrem Liegenden). Sie ist mit abgetragenem sandigschluffigem Feinmaterial der benachbarten Wallmoräne überdeckt. In Anbetracht der Steilheit des Moränenhanges von vorrückenden Gletschern und im Hinblick auf das Klima während des Gletschervorstoßes dürfte der Abtrag des Feinmaterials und damit die Zusedimentierung des Torfes schon während des Gletschervorstoßes begonnen haben. Das obere ${ }^{14} \mathrm{C}$-Datum des Torfes (datiert wurde eine Torfprobe aus der obersten $1-2 \mathrm{~cm}$ mächtigen Lage) kann daher zur Datierung des Gletschervorstoßes, der die benachbarte Wallmoräne schuf, herangezogen werden. Das Ende der Torfbildung, wahrscheinlich infolge Überdeckung mit Feinmaterial, ist nach $1350 \pm 65$ yBP (UZ-2647/ETH-7066) erfolgt. Es ist anzunehmen, daß die Datierung einer noch schmäleren Torflage ein etwas jüngeres Datum ergeben hätte.

Das ${ }^{14}$ C-Alter der Torfbasis $(3590 \pm 70$ yBP, UZ-2649/ ETH-7068) - es wurde ebenfalls eine 1-2 cm mächtige Torflage datiert - belegt, da $\beta$ die Torfbildung vor rund 3600 Radiokarbonjahren begann. Diese Stelle ist mindestens seit jener Zeit, also seit mindestens 3600 Jahren, eisfrei und landfest geblieben. Die seither erfolgten Gletschervorstöße haben demnach das durch den Moränenwall begrenzte Gletschervorfeld seit dem mittleren Subboreal nie mehr «überschritten».

Unter der Wallmoräne legten wir eine weitere Torflage frei, die u. E. am Ort ihrer Bildung liegt (in situ). Zwei ${ }^{14} \mathrm{C}$ Datierungen ergaben Alter von $810 \pm 65$ yBP, UZ-2635/ ETH-6912 und $600 \pm 50$ yBP, UZ-2719/ETH-8097. Aufgrund der vorliegenden Befunde fällt die Mise en place der äußersten Wallmoräne des dreiteiligen Moränenwalls ins frühere Jüngere Subatlantikum. Wenn man bedenkt, daß fünf Wallmoränen bis zum heutigen Gletscherrand beobachtet wurden, ist nicht auszuschließen, daß die äußerste Moräne selber durch einen älteren Glet- 
schervorstoß gebildet und durch einen späteren in ihre heutige Lage verschoben wurde.

Die ${ }^{14} \mathrm{C}$-Daten von Proben der in situ-Torflage unter der Wallmoräne im Aufschluß Gc sind jünger ( $370 \pm 75$ yBP, UZ-2639/ETH-6916, $485 \pm 50$ yBP UZ-2718/ETH8096), während eine schmale, an den Rundhöcker gepreßte Torflage wahrscheinlich nicht am Ort der Torfbildung liegt, sondern beim Gletschervorstoß um $1060 \pm 55$ yBP, UZ-2688/ETH-7576, im Vorfeld abgetragen wurde.
Daraus kann auf einen Gletschervorstoß an der Wende Älteres/Jüngeres Subatlantikum geschlossen werden; außerdem ist anzunehmen, daß die Mise en place der Moräne im Hangenden erst im mittleren Jüngeren Subatlantikum abgeschlossen war.

Ein Vergleich mit Radiokarbondaten von andern Autoren ist, ohne daß man die Lagerungsverhältnisse kennt, mit Zurückhaltung vorzunehmen. Unsere beiden Daten

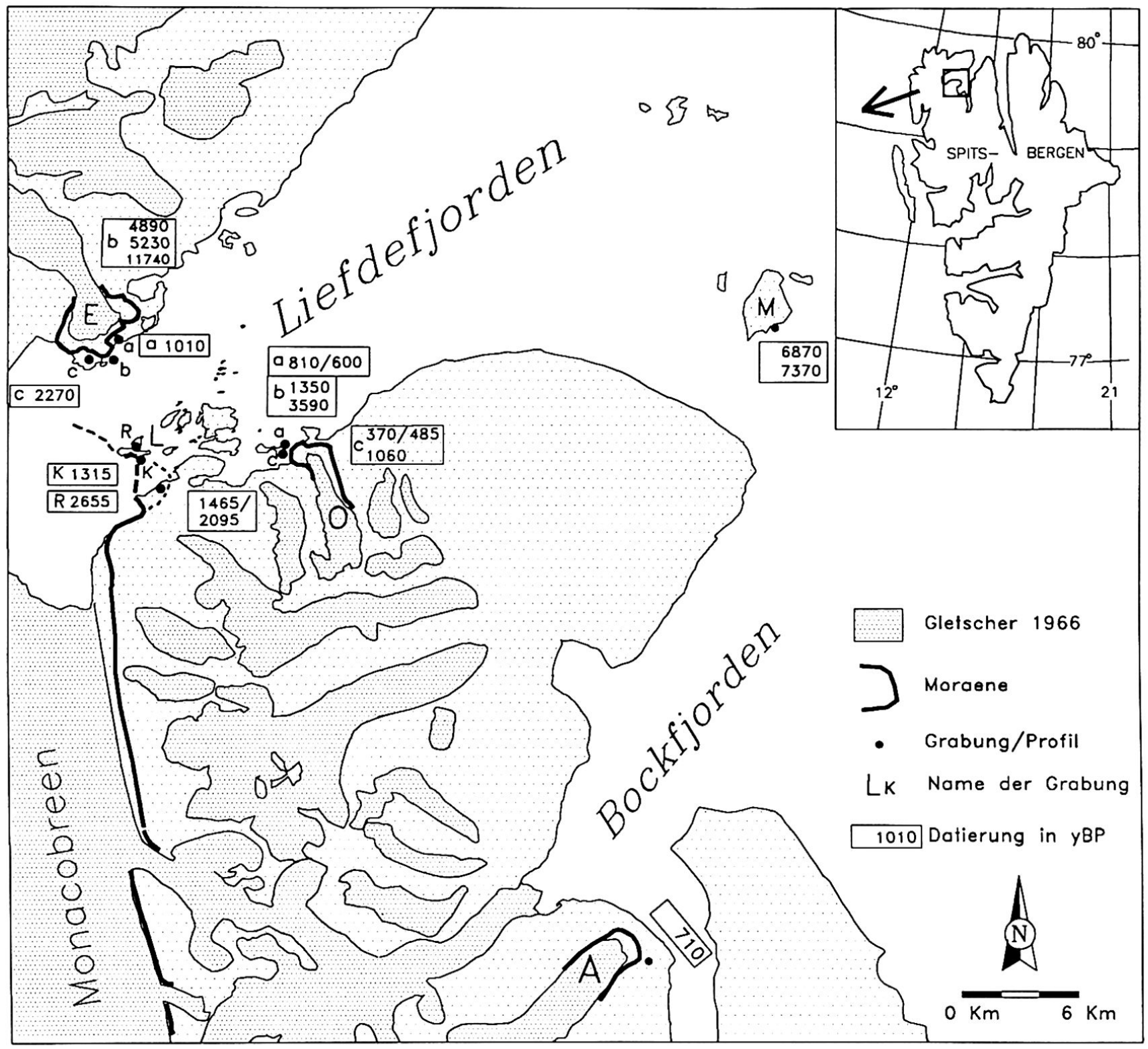

Fig. 2: Im Expeditionsgebiet überraschte uns die große Zahl der beobachteten fossilen organischen Horizonte unter Wallmoränen. Es handelt sich dabei um Torfe oder - in der Mehrzahl der Fälle - um Humus. Die Humushorizonte betrachten wir als Bestandteile von fossilen Böden. Die Auswertung ihrer Makroreste sowie die Pollenanalyse sollen zeigen, ob diese fossilen Böden im Sinne von Klimagunstphasen gedeutet werden können. Die ${ }^{14} \mathrm{C}$-Daten aus Torf stammen beinahe alle von Material aus in situ-Lage, jene der Humushorizonte immer - das organische Material wurde also am Ort seiner Bildung fossilisiert, und sein Radiokarbonalter gibt den Zeitpunkt des Absterbens von Pflanzen wieder.

$L=$ Lerneroyane $M=$ Måkeyoane $A=$ Adolfbreen $E=$ Erikbreen $O=$ Glopbreen 
$-810 \pm 65$ yBP und $1060 \pm 55$ yBP - , mit denen wir den Abschluß eines Gletschervorstoßes (Mise en place der äußersten Wallmoräne) bzw. einen Gletschervorstoß zeitlich einstufen, erfahren vielleicht eine Bestätigung durch Boulten (1979, p. 52). Er zitiert u. a. zwei identische ${ }^{14} \mathrm{C}$-Daten als Belege für Gletschervorstöße: $810 \pm 70$ yBP Hornsund und $1080 \pm 105$ yBP Werenskiölbreen).

\section{Beobachtungen im Bockfjord}

Mit seiner orographisch rechten Seite tangiert der Adolfbreen einen erloschenen Vulkan. Zwischen diesem und der rechten Seitenmoräne ist in einem Bacheinschnitt über dem Anstehenden aus olivinreichem Basalt und unter Moränenmaterial ein fossiler Boden über mehrere Meter aufgeschlossen. Diese Beobachtung bewog uns, an einer andern Stelle, nämlich vor der etwa $15 \mathrm{~m}$ hohen Endmoräne in rund $40 \mathrm{~m}$ ü. M., zu graben. Diese Endmoräne ist der äußerste zu beobachtende Stand. Den Graben trieben wir vom schwach geneigten Hangfuß aus Material, das von der Endmoräne stammt und viele kantengerundete Steine enthält, gegen die steiler ansteigende Moränenaußenseite einige Dezimeter unter die Moräne vor. Die Oberfläche des anstehenden Basaltfelsens im Liegenden ist mechanisch angewittert, sie fällt leicht gegen den Bockfjord ein. Auf den losgewitterten Trümmern, zwischen denen Feinerde mit organischem Material liegt, bereitet sich in gut $0,5 \mathrm{~m}$ Tiefe der obenerwähnte organische Horizont von 3-6 cm Mächtigkeit aus, ein Horizont, den wir als fossilen Boden deuten. Schluffigfeinsandiges, steinfreies anorganisches Material von $15-20 \mathrm{~cm}$ Mächtigkeit deckt ihn zu. Darüber folgt eine kaum $5 \mathrm{~cm}$ mächtige Feinsandlage, die Steinchen enthält. Über ihr liegt, in der Grabung gut $40 \mathrm{~cm}$ mächtig, steinreiches Moränenmaterial, das viele kantige Blöcke enthält. Der organische Horizont zieht unter die Wallmoräne hinein.

Eine Radiokarbondatierung des fossilen Bodens ergab ein Alter von $710 \pm 60$ yBP (UZ-2636/ETH-6913). Wie am Glopbreen ist auch bei diesem Beispiel anzunehmen, daß die Fossilisierung nicht lange nach der Mise en Place des das Gletschervorfeld abschließenden Endmoränenwalls erfolgte. Der zugehörige Gletschervorstoß dürfte im frühen Jüngeren Subatlantikum, im 12. oder 13. Jahrhundert, abgeschlossen worden sein.

Fjordeinwärts vom Adolfbreen liegen Nygaard- und Schjelderupbreen mit prachtvollen Moränenwällen um ihre Gletschervorfelder. Auch diese Moränenwälle werden, wie beim Glopbreen, aus mindestens zwei bis drei Wallmoränenkränzen gebildet. Im Endmoränenwall des Schjelderupbreens liegt eine morphographische Schlüsselstelle: Seine äußerste Wallmoräne ist auf einer Breite von etwa $8 \mathrm{~m}$ von einem Bach - der heute nicht mehr fließt - bis nahe an die Moränenbasis heran ausgeräumt worden. Unmittelbar dahinter, parallel zum äußersten Wall, liegt ein jüngerer Wallmoränenkranz, der den einstigen Bachlauf zudeckt, die ausgeräumte Stelle glet- scherwärts versperrt und somit die weitere Ausräumung des äußersten Wallmoränenkranzes verhindert; $m$. a. W.: es müssen mindestens zwei verschieden alte Gletschervorstöße von etwa gleicher Größenordnung beim Aufbau des das Gletschervorfeld säumenden Moränenwalls beteiligt gewesen sein. Folgt man dem Moränenwall, so können Abschnitte - besonders an seiner Außenflanke beobachtet werden, wo ein jüngerer, interner gelegener Wallmoränenkranz den älteren, äußeren überschüttet hat. Somit können nacheiszeitliche (postglaziale) Moränenwälle in der Arktis aus Material von zeitlich verschiedenen Gletschervorstößen aufgebaut sein, wie dies auch aus den Alpen bekannt ist (FURRER, 1991a).

\section{Der Moränenwall des Erikbreen (FURRER, 1991b)}

An zwei Stellen, wo Rundhöcker aus dem Liegenden des Moränenwalls, der das Gletschervorfeld säumt, auftauchen, wurden Grabungen gegen die Außenseite dieses Moränenwalls vorgetrieben ( $\mathrm{Ea}$ und $\mathrm{Ec}$ ). Dabei sind in jeder Grabung organische Horizonte freigelegt worden, welche die zeitliche Stellung der Mise en place von Wallmoränen bestimmen lassen, die am Aufbau des Moränenwalls beteiligt sind. Bei diesen organischen Horizonten handelt es sich um Humushorizonte von fossilen Böden (fAh). Deren pollenanalytische Untersuchung läßt auf Pflanzengesellschaften schließen, die dem Verband der Silberwurz-Gesellschaften angehören dürften (FURRER, 1991b). Weil Silberwurz-Gesellschaften im Expeditionsgebiet heute in Lagen unter rund $150 \mathrm{~m}$ auftreten, herrschten zur Zeit der damaligen Bodenbildung bei Ea und Ec klimatische Bedingungen, die kaum ungünstiger waren als die heutigen in Küstennähe.

Das ${ }^{14} \mathrm{C}$-Alter (Ea) einer Probe des fAh-Horziontes in $110 \mathrm{~cm}$ Tiefe beträgt $1010 \pm 80 \mathrm{yBP}$ (UZ-2637/ETH6914). Daraus folgern wir, daß die Mise en Place der Wallmoräne am Übergang Älteres/Jüngeres Subatlantikum erfolgte. Auch wenn die Fossilisierung dieses Horizontes im Bereiche unserer Grabung lediglich durch abgetragenes Material der Wallmoräne erfolgt sein sollte - mit unserer Grabung die Basis des Walles allenfalls nicht aufgeschlossen wurde -, ist bei der Nähe, Höhe und Steilheit des Walles nicht anzunehmen, daß der Hangabtrag und damit die Fossilisierung lange Zeit nach der Mise en Place erfolgte. Wir folgern also, daß das ${ }^{14} \mathrm{C}$-Alter dem Zeitpunkt der Mise en Place entspricht.

Die datierte Probe der Grabung Ec, aus gut 1,5 m Tiefe stammend, wurde direkt auf dem Fels gewonnen. Aufgrund des ${ }^{14} \mathrm{C}$-Alters $(2270 \pm 80 \mathrm{yBP}, \mathrm{UZ}-2634 / \mathrm{ETH}-$ 6911) stellen wir die Mise en Place der benachbarten Wallmoräne ins frühe Ältere Subatlantikum. Obwohl auch diese Wallmoräne wie jene von Ea die äußerste Begrenzung des Moränenwalles um das Vorfeld des Erikbreen bildet, ist sie doppelt so alt. Daraus muß gefolgert werden, daß der Endmoränenwall des Erikbreen aus Wallmoränen von mindestens zwei Gletschervorstößen aufgebaut ist. 


\section{Aufschluss Eb}

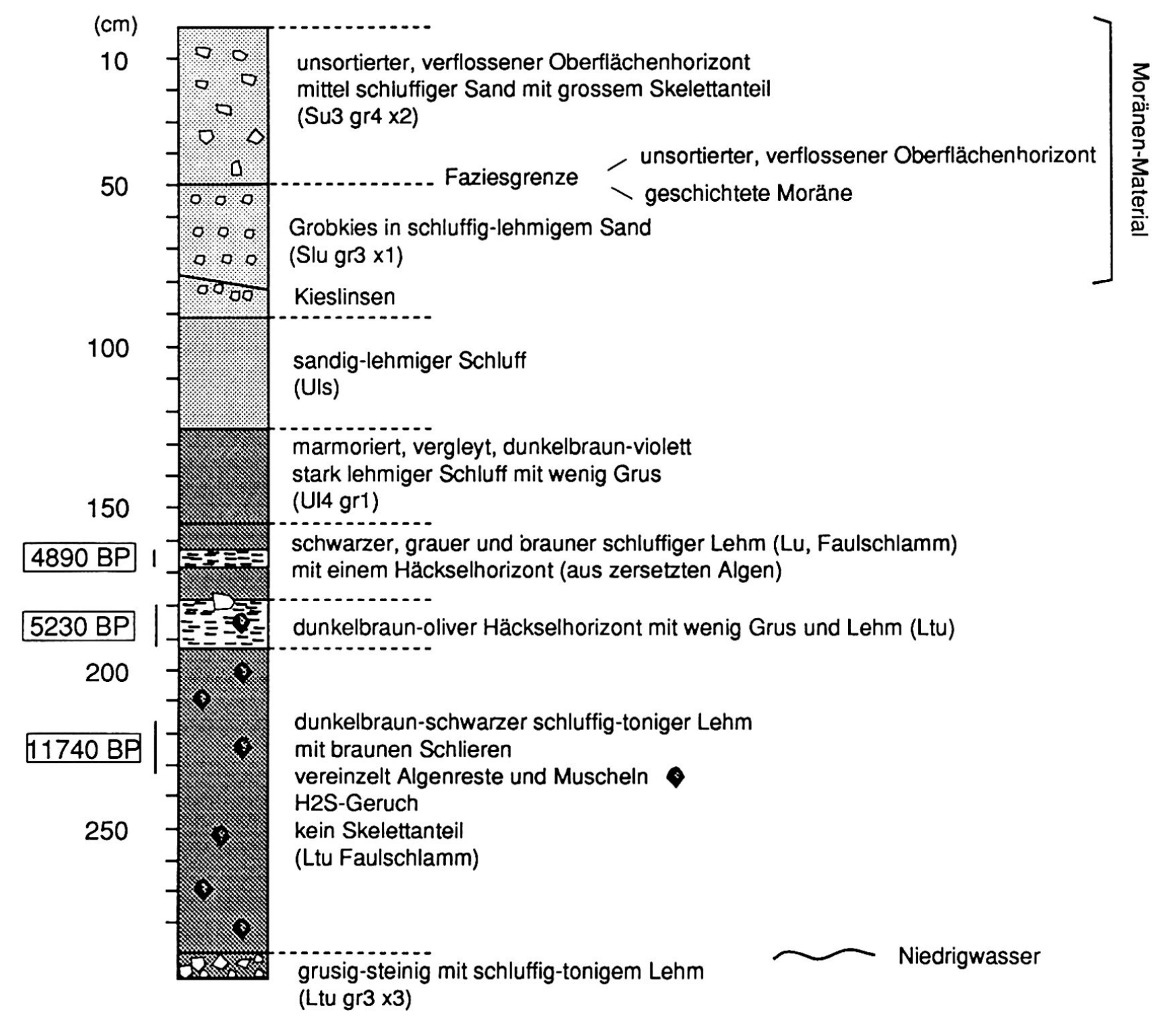

Abgrenzung der Korngrössenklassen und Kurzzeichen gemäss Bodenkundlicher Kartieranleitung

Profilansprache und Korngrössenanalyse: Arbeitsgruppe Prof. Dr. W.-D. Blümel

Geographisches Institut

Universităt Stuttgart

Fig. 3: Das aktive Kliff, Aufschluß Eb. $4890 \pm 75$ yBP (UZ-2643/ETH-7062), $5230 \pm 70$ yBP(UZ-2642/ETH-7061), $11740 \pm 95$ yBP (UZ-2687/ETH-7574). Die Profilbasis liegt im Niedrigwasserbereich. 
Zwischen diesen beiden Grabungen greift die Rückverlegung des aktiven Kliffs an einer Stelle den Moränenwall an (Eb). Das Profil (Fig. 3) zeigt eindrücklich, daß der Moränenfuß in $50-80 \mathrm{~cm}$ Profiltiefe aufgeschlossen ist, der Fuß des Moränenwalls also an seinem äußersten Rand schon etwas abgetragen wurde. Darüber ist disloziertes Moränenmaterial, das vom Moränenwall stammt, aufgeschlossen.

Die Moräne kann frühestens am Übergang vom Atlantikum zum Subboreal an der Profilstelle abgelagert worden sein (oberstes ${ }^{14} \mathrm{C}$-Datum). Diese Profilstelle mit dem Radiokarbonalter von 11740 Jahren, direkt am Ufer gelegen, läßt den Schluß zu, daß der Erikbreen sein heutiges Vorfeld während der gesamten Nacheiszeit, also seit dem Übergang Jüngere Dryas/Präboreal vor 10200 Radiokarbonjahren, nie mehr überschritten hat. Diese Feststellung gilt, sofern tatsächlich im Profil keine Schichtlükke, wo Moränenmaterial abgetragen wurde, vorhanden ist. Damit ist eine Parallele zur Geschichte der Alpengletscher aufgedeckt: Auch jene haben im Postglazial die Dimension der Gletschervorstöße der jüngsten Vergangenheit (in den Alpen: der Nacheiszeit, also ab 16. Jahrhundert) nie wesentlich überschritten (FURRER, 1991a). Außerdem muß die Profilstelle schon im Alleröd eisfrei gewesen sein. Diese Feststellung wird durch MANGERUD und SVENDSEN (1990, S. 267) gestützt, welche die Enteisung im Linnévatnet um 12500 yBP ansetzen. Die Frage der jüngsten spätglazialen Vorstöße während der jüngeren Dryaszeit - in den Alpen die Egesenvorstöße über das postglaziale Ausmaß hinaus - ist offen: In unserem Profil läßt sich keiner nachweisen, ein Resultat, zu welchem auch MANGERUD und SVENDSEN (1990, S. 267 f.) gelangen, während BOULTEN (1979, S. 51 f.) einen gegenteiligen Standpunkt vertritt.

Die Muschelschalen der Profilbasis bilden Gegenstand besonderer Untersuchungen: Beispielsweise ist Mytilus edulis im Liefdefjord bisher (1990) nicht lebend gefunden worden, sie lebt heute im W und S Spitzbergens. Besonders interessant ist die Kleintierfauna, die sich in dem die Schalen umgebenden Material befindet: Sie enthält Foraminiferen, Ostracoden, Bryozoen und Seeigelstacheln. Für die Ostracodenfauna kann schon gesagt werden, daß sie nur zum Teil mit der rezent im Untersuchungsgebiet lebenden Fauna übereinstimmt. Dieses geborgene Material wird von unserem Expeditionskameraden, Professor G. HARTMANN, Zoologe an der Universität Hamburg, in der Hoffnung, Hinweise auf ökologische Parameter zu gewinnen, bearbeitet.

Weil die beiden datierten Häckselhorizonte unter dem Meeresspiegel abgelagert wurden, muß Transgression bis mindestens zum Ende des Atlantikums (4800 yBP) geherrscht haben, seither dominiert an dieser schon vor dem Alleröd enteisten Lokalität Heraushebung.

\section{Måke-Inseln}

Auf der südlichsten, der Store Måke Oya, an ihrer festlandnächsten Ecke, haben wir Ende Juli unmittelbar am
Ufer, knapp über dem Hochwasserbereich und im Anschluß an den schmalen Strandgeröllstreifen, ein Torfprofil gegraben. In $60 \mathrm{~cm}$ Tiefe wurde der Permafrost erreicht, er fällt durch Linsen aus blankem Eis auf. $11 \mathrm{~cm}$ tiefer endet der nur wenig zersetzte Torf, in seinem Liegenden tritt grober Kies auf. Das Radiokarbonalter der Torfbasis in $71 \mathrm{~cm}$ Tiefe, knapp über dem Hochwasserniveau gelegen, beträgt $7370 \pm 80$ yBP (UZ-2648/ETH7067), jenes einer Torfprobe unmittelbar über dem Permafrost in 59-60 cm Tiefe $6870 \pm 70$ BP (UZ-2689/ ETH-7577). Diese Stelle der Insel ist folglich seit 7370 Radiokarbonjahren eisfrei und - aufgrund der Pollenanalyse der Torfprofilsäure zu schließen - landfest.

\section{Lerneroyane und Monacobreen}

\section{Die Neuzeit}

Die Kalbungsfront des Monacobreen liegt heute etwa $6 \mathrm{~km}$ von der westlichsten Lernerinsel entfernt, 1966 waren es, nach Flugaufnahmen beurteilt, etwa $4 \mathrm{~km}, 60 \mathrm{Jah}$ re früher, also 1906/07 - nach der Karte von Isachsen rund $2 \mathrm{~km}$. Entsprechend diesem Dokument war der Idabreen, der westliche Nachbar vom Erik, dem Monacobreen nicht mehr tributär. Von zwei noch größeren Ausdehnungen des letzteren zeugen eine west-östlich verlaufende, unmittelbar über einem Marmorkliff gelegene, ins Meer hinausstreichende Wallmoräne auf der westlichsten Lernerinsel (FURRER, 1991b) sowie eine intern gelegene, unmittelbar benachbarte kleinere, die noch Toteis besitzt. Letztere läßt sich mit der rechten Seitenmoräne, einem langgezogenen, mehrfach gegliederten Moränenwall des Monacobreen - dessen nördliches in der Karte gebogenes Ende ebenfalls Toteis enthält auf dem Festland verbinden (Fig. 2, dick gestrichelt). Bei diesem Stand dürfte es sich um den neuzeitlichen Hochstand handeln.

Mit dem Rückgang der vergletscherten Fläche ging auch eine Veränderung der Solifluktionstätigkeit einher: Während unserer Expeditionszeit ließen sich aktive Solifluktionsvorgänge und Strukturböden erst ab etwa $500 \mathrm{~m}$ ü. M. beobachten, während in Küstennähe dieser Formenschatz trocken und teilweise mit Vegetation überwachsen ist. Das war - zumindest im Kongsfjord, wo die Gletscher inzwischen auch kleiner geworden sind - Mitte der 50er Jahre noch nicht der Fall (FURRER. 1959).

\section{Vorstoß im jüngeren Älteren Subatlantikum}

Aufder Südseite der Insel, in $1 \frac{2}{\mathrm{~m}}$ bis in etwa Mannshöhe über dem Meeresspiegel, ist unmittelbar über dem Kliff, auf einer Strecke von über $100 \mathrm{~m}$, auf dem Fels und unter dem größeren, äußeren Wall ein bis zu $10 \mathrm{~cm}$ mächtiger Humushorizont aufgeschlossen. Die Radiokarbondatierung einer Probe (LK) aus dem obersten Abschnitt dieses Horizontes ergab ein Alter von $1315 \pm 100$ yBP (UZ- 
2638/ETH-6915). Wir schließen daraus auf einen Vorstoß im jüngeren Älteren Subatlantikum und folgern, da $B$ die Kalbungsfront des Monacobreen damals auf der Höhe dieser Lernerinsel lag, ähnlich wie beim neuzeitlichen Hochstand. Zur Zeit des mit Hilfe der Grabung Ea belegten Hochstandes des Erikbreen war daher auch der Monacobreen größer, etwa $6 \mathrm{~km}$ länger als heute; die beiden floßen aber nicht zusammen.

\section{Bodenbildung im frühen Älteren Subatlantikum/Subboreal}

Aufgrund einer Grabung, etwa $150 \mathrm{~m}$ außerhalb der erwähnten Wallmoräne, um 13 m ü. M. - also höher als die Basis der Wallmoräne und höher als die datierte organische Lage in deren Liegendem - schließen wir auf eine noch größere Ausdehnung des Monacobreen (FURRER, 1991b). Weil wir keine dazugehörende Wallmoräne finden, ist sie in ihrem Ausmaß nicht bestimmbar. In der Grabung (LR) durch bewachsene Grundmoräne liegt in $80 \mathrm{~cm}$ Tiefe Humus auf einem Rundhöcker. Aus seiner Lagerung - in seiner Mächtigkeit stark schwankend, besonders unter Blöcken und in Felsnischen der Rundhöckeroberfläche gut erhalten - gewinnt man den Eindruck, daß beim Vorstoß des Gletschers der Humushorizont gestört wurde. Sein Alter ( $2655 \pm 65$ yBP) weist zusammen mit dem Alter des fAh der Grabung Ec - auf eine noch ältere Bodenbildung und die überdeckende Moräne auf frühere Hochstände von Monaco- und Erikbreen im Älteren Subatlantikum hin. Dieses Resultat wird durch eine Arbeit von russischen Kollegen gestützt, die aufgrund einer Rekonstruktion der Vegetationsentwicklung im Subboreal eine Erwärmung mit einer Feuchtzunahme beobachten und am Übergang Subboreal/Subatlantikum eine kurzfristige Abkühlung postulieren (SEREBRJANNYJ et al., 1984). Aus der Lage der Grabung ergibt sich, daß die südlichste Lernerinsel damals vielleicht mit Ausnahme ihrer höchsten Erhebung - unter Eis lag.

Die kräftig entwickelte, an den Verhältnissen im Liefdefjord gemessen sehr großflächig ausgebildete Humuslage $(1315 \pm 100 \mathrm{yBP}$, Probe LK) im Liegenden der Wallmoräne muß im Älteren Subatlantikum gebildet worden sein: Der Monacobreen schmolz von seiner äußeren Lage im frühen Älteren Subatlantikum hinter die Lernerinsel zurück, bevor der Humushorizont gebildet wurde und der erneut vorstoßende Monacobrenn die Wallmoräne auf ihr ablagerte. Das Ältere Subatlantikum erscheint somit als Phase einer Klimaverbesserung, an seinem Anfang und an seinem Ende charakterisiert durch Klimaungunst mit Gletschervorstößen.

\section{Bodenbildung im mittleren Älteren Subatlantikum}

Das eben beschriebene Ergebnis wird durch eine $10 \mathrm{~m}$ lange Grabung auf dem gegenüberliegenden Festland, auf einer Terrasse, etwa $5 \mathrm{~m}$ ü. M., erhärtet. Eine Grund- moräne mit vielen kantengerundeten, gekritzten Steinen liegt auf einem fossilen Boden, in dessen Liegendem polierter Fels ansteht. Aufgrund von zwei datierten Proben aus $130 \mathrm{~cm}$ Tiefe $(2095 \pm 65$ yBP UZ-2641/ETH-7060 und $1465 \pm 55$ yBP UZ-2721/ETH-8098) beurteilt, wurde auch dieser Boden, dessen Lagerung teilweise gestört ist, im Älteren Subatlantikum gebildet. Der nachfolgende Vorstoß fällt ins jüngere Ältere Subatlantikum. Die zugehörige Grund- und die große Wallmoräne auf der Insel gehören zum selben Vorstoß des Monacobreen (Fig. 2, dünn gestrichelt). Die dazugehörige Gletscherausdehnung dürfte die neuzeitliche nur wenig übertroffen haben, Ida- und Erikbreen flossen nicht mit dem Monacobreen zusammen.

\section{Pollenanalysen}

Die Radiokarbondatierungen lassen Aussagen über den Zeitpunkt von Gletschervorstößen und Phasen mit geringeren Gletscherausdehnungen zu. Zusätzlich sollten pollenanalytische Untersuchungen des geborgenen fossilen organischen Materials Hinweise zur Vegetation und zu den klimatischen Bedingungen während der Sedimentation/Torfbildung ermöglichen. In Figur 4 sind als Beispiel die Polleninhalte von einem rezenten (Probe $G$ rez) und fünf fossilen organischen Horizonten graphisch dargestellt. Die Oberflächenprobe $G$ rez ist in einem nur wenige Meter von der Grabung Ga entfernt liegenden Moor gewonnen worden.

Mit Ausnahme der Profile Eb und Måke, die mehrere Dezimeter mächtig sind, wurde in Folge der geringen Mächtigkeit der fAh-Horizonte jeweils nur eine Probe pro Aufschluß analysiert. Leider waren die Proben von Eb und Måke praktisch pollenfrei, so daß die Ergebnisse unserer palynologischen Untersuchungen sich nur auf Einzelanalysen von geringmächtigen Horizonten stützen und somit nur spärliche Aussagen zur Klima- und Vegetationsgeschichte im Expeditionsgebiet zulassen.

Der Pollengehalt und der Erhaltungszustand der Pollen ist allgemein eher schlecht. Bei fast allen pollenhaltigen fAh-Proben ist der hohe Anteil von Salix polaris auffällig. In sämtlichen Proben konnten zudem Pollen von Dryas octopetala und Cassiope tetragona nachgewiesen werden. Silberwurz-Gesellschaften (Dryadion octopetalae, nach RONNING 1965 und HARTMANN 1980) wie beispielsweise das Salici polaris-Drydetum und das Cassiopo tetragonaeDrydetum sind heute aber im Untersuchungsgebiet nur in den unteren Lagen (zwischen 0 und $150 \mathrm{~m}$ ü. M.) in Küstennähe anzutreffen. Zudem zeigt ein Vergleich mit Rezentproben, daß sich in den von uns analysierten, von Gletschern überfahrenen Bodenbildungen Vegetationsgesellschaften abzeichnen, die heute nur in Küstennähe gedeihen.

Die klimatischen Bedingungen dürften demnach zu den Zeiten der nachgewiesenen ehemaligen Bodenbildungen mindestens nicht ungünstiger als heute im Küstenbereich gewesen sein. 

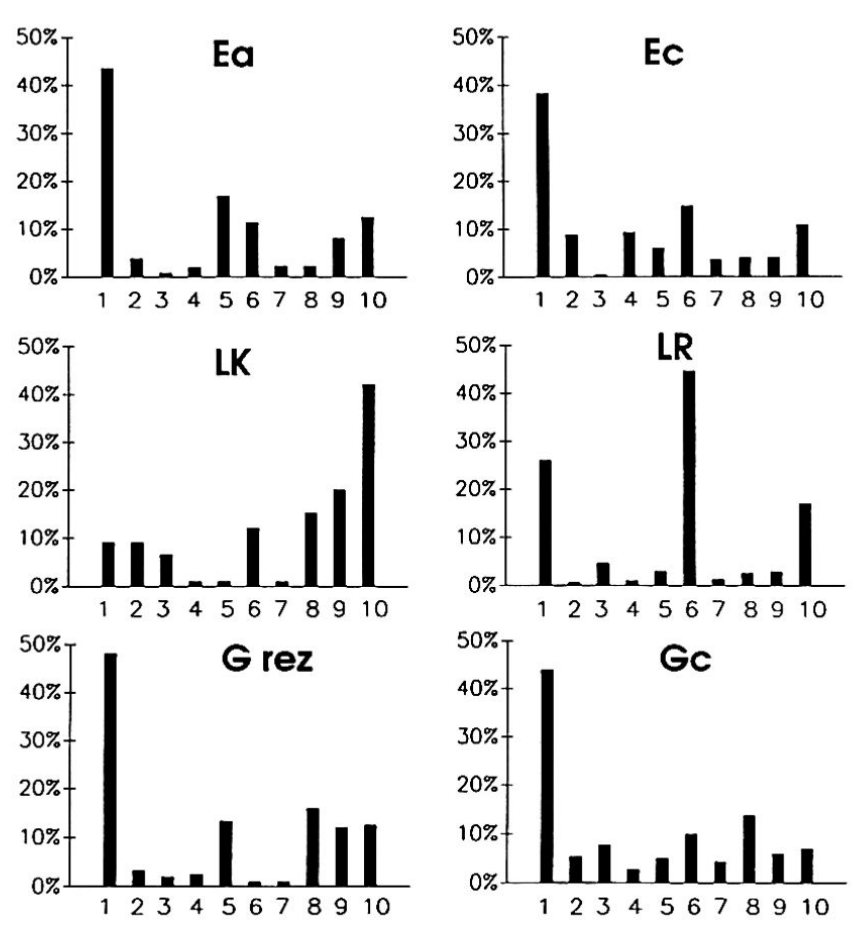

Fig. 4: 1 Salix polaris, 2 Cassiope tetragona, 3 Dryas octopetala, 4 Saxifraga oppositif.-Typ, 5 Saxifraga caespitosa-Typ, 6 Saxifraga nivalis-Typ, 7 Caryophyllaceae, 8 Poaceae, 9 Cyperaceae, 10 Varia/indet. (unbestimmte/unbestimmbare Krautpollen). Es sind nur die 10 wichtigsten Taxa/Kategorien dargestellt. Pollensumme $(100 \%)=$ alle gezählten Pollen ohne Cyperaceae. Bei der routinemäßig in 250 facher VergröBerung erfolgten Analyse wurde wenn möglich jede Probe auf mindestens 250 Pollen ausgezählt. (LK und LR: Analyse Conradin Burga. Beide Proben enthalten in großer Zahl mikroskopisch kleine, feinzersetzte Pflanzenreste aller Art).

\section{Literatur}

BOULTON, G. S. (1979): Glacial history of the Spitsbergen archipelago and the problem of a Barents Shelf ice sheet; in: Boreas, Vol. 8, Nr. 1, S. 31-57, Oslo.

FURRER, G. (1959): Untersuchungen am subnivalen Formenschatz in Spitzbergen und in den Bündner Alpen; in: Geographica Helvetica, Heft 4, S. 277-309.

FURRER, G. (1991a): 25000 Jahre Gletschergeschichte; Neujahrsblatt der Naturf. Ges. Zürich, Heft 5.

FURRER, G. (1991b): Zum Alter von Wallmoränen um das Vorfeld des Erikbreen und auf der westlichsten Lernerinsel ein Beitrag zur Gletschergeschichte des Liefdefjords (Erste Ergebnisse der Geowissenschaftlichen Spitzbergenexpedition 1990 des Teilprojektes "Gletscher und Moränen als Klima- und Datierungsphänomen»); Festschrift Karl Brunnakker, Geol. Inst. Univ. Köln.

HARTMANN, H. (1980): Beitrag zur Kenntnis der Pflanzengesellschaften Spitzbergens; in: Phytocoenologia, 8: S. 65-147.

MANGERUD, J. \& SVENDSEN, J. I. (1990): Deglaciation chronology inferred from marine sediments in a proglacial lake basin, western Spitsbergen, Svalbard; in: Boreas, Vol. 19, S. $249-272$, Oslo.

RONNING, O. (1965): Studies in Dryadion of Svalbard; in: Norsk Polarinst., Skr. 134.

SEREBRJANNYJ, L. R.; TISKOV, A. A.; MALJASOVA, E. S.; SOLOMINA, O. N.; IL'VES, E. O. (1984): Rekonstruktion der Vegetationsentwicklung in der Hohen Arktis; in: Izvestija Akademii Nauk SSSR, serija geograficeskaja, H. 6, S. 75-84. (Übersetzung von Burkhard Frenzel). 